\title{
Design and Implementation of Error Remote Calibration System Related to Gateway Energy Measuring Devices
}

\author{
Xuhui Shu ${ }^{1, a^{*}}$, Wei $\mathrm{Bi}^{2, \mathrm{~b}}$, Shengqing $\mathrm{Wei}^{3, \mathrm{c}}$, Dengping Tang ${ }^{4, \mathrm{~d}}$ and $\mathrm{Kai} \mathrm{H}^{5, \mathrm{e}^{*}}$ \\ ${ }^{1,3}$ State Grid Hubei Electric Power Company, Hubei, China \\ ${ }^{2,4}$ Electric Power Research Institute, State Grid Hubei Electric Power Company, Hubei, China \\ ${ }^{5}$ Beijing VIS Energy Technology Co., Ltd., Wuhan, Hubei, China \\ axuhui_sgg@126.com, bweibi_sgg@126.com, 'shengqing_sgg@126.com, \\ 'dengping_sgg@126.com, ${ }^{\mathrm{e}} \mathrm{hkai@visenergy.cc}$
}

\begin{abstract}
Keywords: Gateway Energy Measuring, Real-time Monitoring, Dynamic Analysis of Data, Error Remote Calibration

Abstract. Substation is always equipped with electric energy metering system which does not have the function of error remote calibration. In this paper, we introduce the error remote calibration system related to energy measuring devices to achieve two functions: on the one hand is to minimize the relevant losses by acquiring and processing the data of voltage transformers and current transformers, real-time monitoring the running status of voltage and current in secondary circuit and measuring the error of watt-hour meters; on the other hand, by means of the remote monitored data which is collected by the mainstream of communications to manage and analyze the data, alarm timely when problems happen and realize the automation management of energy metering devices.
\end{abstract}

\section{Introduction}

Energy metering devices include various types of watt-hour meters, measuring voltage and current transformers in secondary circuits and energy metering cabinets (boxes) etc. According to the regulations in "DL / T 448-2000 Energy Metering Device Technical Management Rules": the running energy metering devices have been divided into five classes(I, II, III, IV, V ) according to the significance of metering objects and the metering amount of energy. Class I refers to the energy metering devices which have the average monthly power consumption of 5,000,000 kWh and above or transformer capacity is $10000 \mathrm{kVA}$ and above or generator's capacity is $200 \mathrm{MW}$ and above or the energy metering devices in energy exchange points of power grid corporation and power supply energy metering points between provincial power grid corporation and their power suppliers. Thus gateway energy metering devices all belong to the class I and are usually installed in the substations[1,2].

Gateway metering devices is used to measure of the flow and size of power in gateway boundaries, and the power energy they records can be regarded as the basis data of technical and economic indicators. Meanwhile, they are the key to ensure the healthy operation of the power market, and the accuracy of measurement is directly related to the economic and social benefits of power supply enterprises[3,4].

\section{Overall Analysis and Design of the System}

Energy metering devices' remote calibration and monitoring system has the following characteristics:

(1)The system requires high real-time performance: the devices may fail in any time during the testing process, and if that is not timely found, the enterprise will get great losses, so we need to monitor and record devices' running status in 24 hours to check problems and alarm timely.

(2) The system has high security requirements: in sampling process, the energy metering staff often need to do high-voltage power line work, which will undoubtedly bring hidden danger to the operator; meanwhile, the mishandling operation of energy metering staff can result in great losses to the related 
devices. Therefore, the utilization of the system must be on the premise of ensuring the safety of staff and reducing the harm to devices.

(3) The system has high reliability requirements: in order to ensure the healthy and effective running of power enterprises, energy metering devices' remote calibration and monitoring system must have high reliability, which mainly refers to the accuracy, stability, convenience of the metering devices and the maintainability of the system.

Functional analysis of field detection devices. In order to avoid energy metering devices' shortcomings of monitoring the error of electric meters and the voltage and current in secondary circuit ineffectively and to ensure the safety and efficiency of the power sector, field detection devices must have the following functions:

(1) Having the function of achieving the power instantaneous parameters by locally and remotely online monitoring, including reactive errors and active errors of watt-hour meters and other metering devices, error oversize of electric meters, pressure drop gauge and overloading or light-loading of secondary loads. When detecting electric meters in specific detection period, field detection devices can automatically read the transient parameters, and automatically saved to monitor electric meters' running status and errors and alarm timely when various abnormalities happen.

(2) Having the function of judging the running status of PT and CT in secondary circuit accurately. Using the parameters' real-time values monitored by the system and the data monitored when outage happens to monitor the actual loads and voltage drop of CT and PT in secondary circuit, and then we can see if the monitoring results meet the running requirements.

(3) Having the function of automatically and manually metering the error and standard deviation of energy metering devices and saving the records and detection results. Making full use of the precision changing trends of electric meters and the monitoring data to show the vector diagrams of voltage and current, and then it can meet wiring field checking requirements.

(4) Having the function of automatically diagnosing. By means of the analysis of data in related reports to automatically diagnose the current status of the various functional modules of the system and warn operators before faults happens through ALARM LEDs in the front panel.

Functional analysis of master station management center. Master station management center is a core component of the system, and it can remotely control the field detection devices, including the management of data obtained by devices and electric parameters used for tests and the calibration of remote monitoring results. So the design of master station management center must have the following functions:

(1) Having the function of managing the collected data. Generating report based on the online monitoring data and analyzing the trend graph of related parameters to get ready for the subsequent data analysis. By regularly analyzing of the data for each monitoring sites in the reports and the data of alarming statistics to facilitate data management of the system, and lay the ground for office automation.

(2) Having the function of use privilege allocation which can be able to manage the privileges of different consumers according to the customer's level. For example, the customers in higher level can manage the customers in lower level including assigning running functions to the customers in lower level. Meanwhile, different levels of customers can view the data stored in WEB based on the running privileges.

(3) Having the function of remotely controlling the field energy metering devices by setting the following parameters: the number of electric meters, pulse constant, wiring, the name of electric lines, the start select of cycle detections, cycle detection time, alarm limits, etc.

To achieve the above requirements, we need to use the current advanced network communication technology which is compatible with the mainstream communication modes, such as: Ethernet, RS232 / RS485, GSM / INTERNET / LAN / PSTN and telephone network to make the network facilitate different occasions. Meanwhile, making full use of Chinese operation interfaces for background management software and modularizing the design schemes to improve the reliability of the system. 


\section{Overall Structure of Error Remote Calibration System}

The system has a layered structure, and the entire system is divided into the following three layers: field monitoring and calibration devices, communication network and master station management centers. The diagram of system's overall structure is shown in Fig.1.

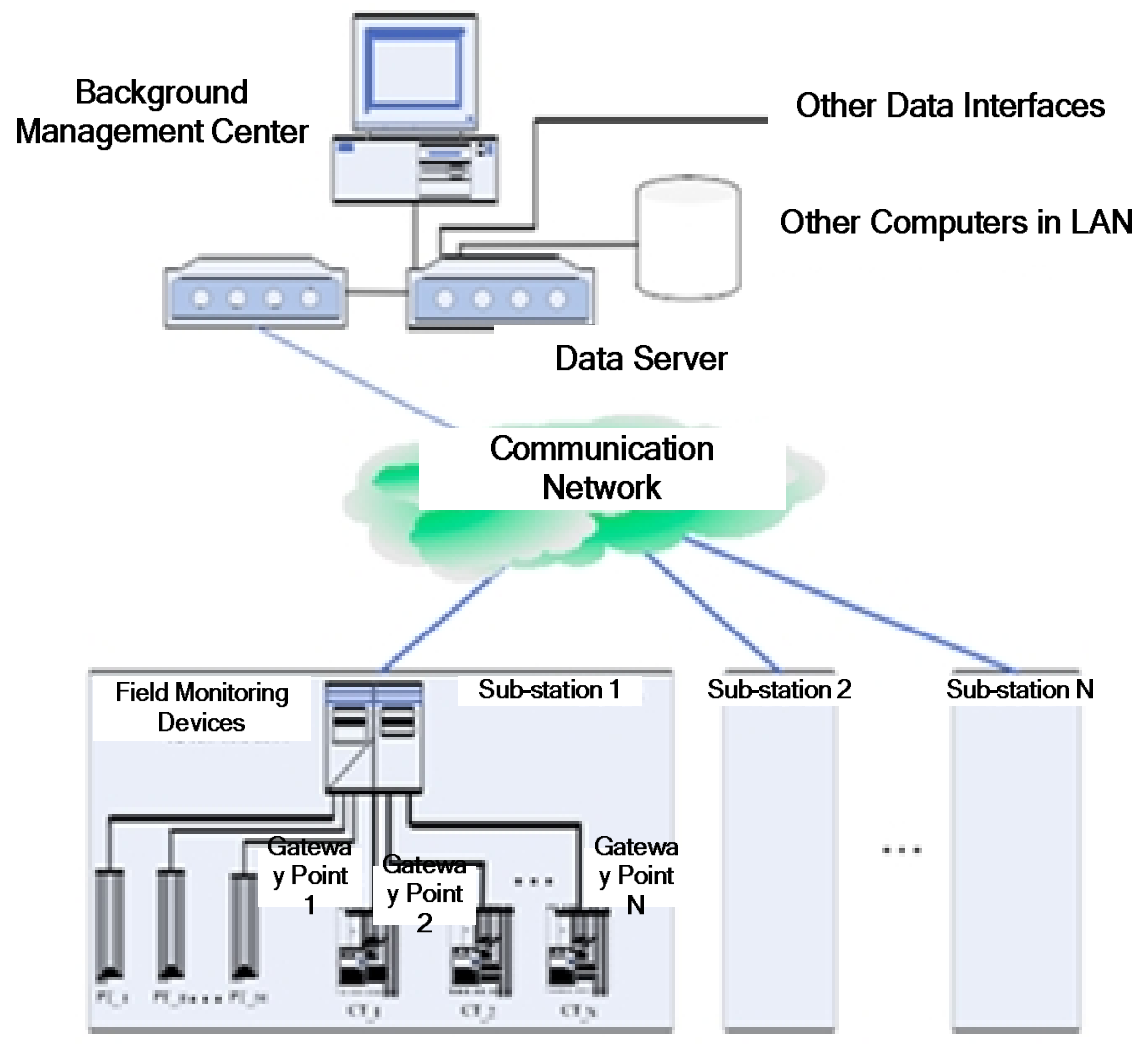

Fig.1 Overall Structure of Error Remote Calibration System Related to Gateway Energy Measuring Devices

(1) Field data collection layer

Installing the multi-function electric meters in the switchgear circuits, or centrally in the measurement and control panels for real-time data acquisition, and then transmitting the data to the power collection terminals via the communication interfaces. Energy information for each metering point are all the collecting targets of the collection terminals, and after the data's acquisition, the collection terminals will comprehensively manage the data. At the same time, energy collection terminals are also responsible for the transmission, forward and order execution to make the master stations have remote maintenance function.

(2) Communication network layer

This layer is the connection layer of master layer and field data collection layer, and is responsible for the communication connection between the upper and lower layers, data collection, data conversion, data transmission, protocol conversion and command conversion to ensure a large number of real-time data collection can be fast transmitted and improve master station layer's comprehensiveness, accuracy and betimes in information acquisition.

(3) Master station layer

This layer is mainly composed of network system, WEB application server, collection server, data server, external auxiliary devices, etc. Meanwhile, it is the information collection and management center of energy metering line monitoring system and can not only obtain and manage field terminal information by GPRS, PSTN, CDMA, but also comprehensively process the related data.

Every power plant or substation which has gateway metering points only need one field monitoring and calibration device to achieve the function of online monitoring and remote calibrating for all the metering devices. Meanwhile, the master station makes full use of communication network to collect 
the data of field devices and utilizes web browser to collect the data which are manually input. Then by carding and analyzing the data to get data reports and trend curves.

\section{Conclusion}

In order to meet the new requirements of gateway metering system's error detection, this paper provides a low-cost solution which is based on the existing error remote calibration system related to gateway energy measuring devices and is economic and practical. By executing the solution, error and metering problems of gateway metering systems can be effectively solved and energy metering devices can be automatically managed. In addition, the maintainability, reusability, scalability of gateway error detection module can be greatly improved. The error remote calibration system this paper proposed can also be used in other metering systems and electric information collection system, and has a broad application prospects.

\section{Acknowledgements}

This work is supported by Science and Technology Projects of State Grid Hubei Electric Power Company ( Study of Intelligent online monitoring and Condition Management System of Gateway Electrical Energy Metering Device ).

\section{References}

[1] Wang Zhongbing, The design and Implementation of Gateway Meters Field Monitoring and Remote Calibration System, Cheng Du, 2013.

[2] Hao Junfeng, Research on Remote Energy Metering Devices' Calibration and Monitoring Systems, Bei Jing, 2012.

[3] Liu Juncheng, Jiao Li, Markrt Status and Development of Power Quality Monitoring Technology, Bei Jing, 2014.

[4] Zhao Qingsong, Research on electric meter calibration and error adjustment mode, Ha Erbing, 2009. 